\title{
Vlasov simulation of Langmuir wave packets
}

\section{T. Umeda}

Solar-Terrestrial Environment Laboratory, Nagoya University, Nagoya, Aichi 464-8601, Japan

Received: 14 May 2007 - Revised: 3 August 2007 - Accepted: 18 October 2007 - Published: 25 October 2007

\begin{abstract}
Amplitude modulation and packet formation of Langmuir waves are commonly observed during a nonlinear interaction between electron beams and plasmas. In this paper, we briefly review the history of Langmuir wave packets as developed by recent spacecraft observations and computer simulations. New one-dimensional electrostatic Vlasov simulations are performed to study their formation processes. It is found that the formation of Langmuir wave packets involves both an incoherent turbulent process and a coherent nonlinear trapping process. Existence of cold ions does not affect nonlinear processes of the weak-electron-beam instability in which the ion distribution is hardly modified by the excited Langmuir wave packets.
\end{abstract}

\section{Introduction}

Electron-beam-plasma interactions are one of the most fundamental processes in space plasmas. It is well known that electron beam instabilities develop into nonlinear waves and turbulence. Electron phase-space-density holes (e.g. Berk and Roberts, 1967) or electrostatic solitary waves (Matsumoto et al., 1994) are coherent nonlinear electrostatic structures, while harmonic Langmuir waves and Langmuir wave packets have an incoherent quasi-power-law wavenumber spectrum which indicate a turbulent feature (Yoon et al., 2003; Gaelzer et al., 2003; Umeda et al., 2003; Umeda, 2006; Silin et al., 2007). The present study is aimed at the generation of amplitude-modulated Langmuir waves and Langmuir wave packets which is called a Langmuir turbulence in space plasmas.

The amplitude modulation and packet formation of Langmuir waves were commonly observed in self-consistent kinetic simulations of electron beam instabilities (Muschietti et al., 1995, 1996; Akimoto et al., 1996; Matsukiyo et al., 2004; Usui et al., 2005; Umeda, 2006; Silin et al., 2007). Strongly modulated waveforms and packets of Langmuir

Correspondence to: T. Umeda

(umeda@stelab.nagoya-u.ac.jp) waves were also observed in various regions of the magnetosphere, such as in the auroral ionosphere (Ergun et al., 1991; Stasiewicz et al., 1996; Bonnell et al., 1997; Pottelette et al., 1999; Lizunov et al., 2001), in the solar wind (Gurnett et al., 1993; Bale et al., 1996; Kellogg et al., 1999b), in the electron foreshock region (Kellogg et al., 1996, 1999a; Soucek et al., 2005), and in the magnetotail (Kojima et al., 1997).

Several nonlinear theories were adopted to explain mechanisms for the amplitude modulation and packet formation of Langmuir waves. The first is the parametric decay of Langmuir waves into ion acoustic waves (e.g. Cairns and Robinson, 1992; Cairns et al., 1998; Pottelette et al., 1999; Matsukiyo et al., 2004; Soucek et al., 2005) or lower hybrid waves (e.g. Stasiewicz et al., 1996; Bonnell et al., 1997; Lizunov et al., 2001). Note that Cairns et al. (1998) demonstrated that electron-beam-driven Langmuir wave packets observed in the magnetosphere lie well outside the region of parameter space for which the modulational instability can proceed.

For the Langmuir decay instability, the amplitude of primary Langmuir waves must be high enough to modify ion distributions. The observations in the magnetosphere have shown that the amplitude of Langmuir wave packets sometimes exceeds several hundred $\mathrm{mV} / \mathrm{m}$. The observed frequency spectra also show apparent double peaks around the electron plasma frequency with a small low-frequency component (Kellogg et al., 1996; Bale et al., 1996; Soucek et al., 2005), which indicates the Langmuir decay instability. On the other hand, The GEOTAIL spacecraft observation in the magnetotail has shown that the amplitude of Langmuir wave packets is several hundred $\mu \mathrm{V} / \mathrm{m}$, which is too small for the Langmuir decay instability. The computer simulations have also demonstrated that amplitude-modulated Langmuir waves with a very small amplitude can be generated during a very-weak-electron-beam instability without ion dynamics (Akimoto et al., 1996; Usui et al., 2005; Umeda, 2006; Silin et al., 2007), which is consistent with the GEOTAIL observation. These observations and simulations suggest that Langmuir wave packets with a small amplitude

Published by Copernicus Publications on behalf of the European Geosciences Union and the American Geophysical Union. 
(A)

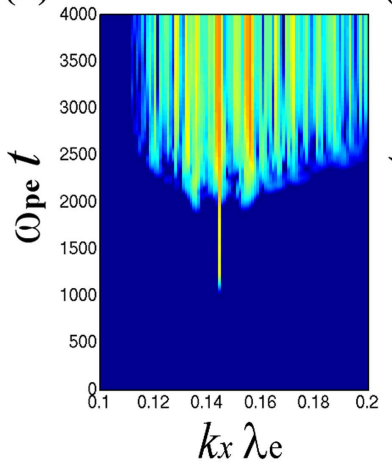

(B)

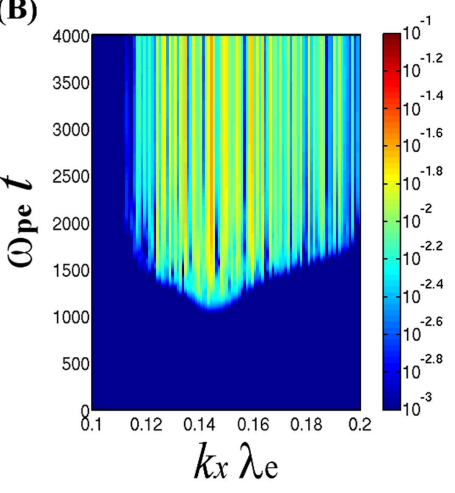

Fig. 1. Time evolution of the wavenumber spectrum for Runs A and B. The wave intensity is normalized by $m_{e} \omega_{p e} V_{t e} / e$.

and a large amplitude are generated by different mechanisms with small amplitude packets generated by electron dynamics while large amplitude packets likely involve ion dynamics as well.

A possible mechanism for the formation of smallamplitude Langmuir wave packets is the "kinetic localization" (Muschietti et al., 1995, 1996), in which Langmuir waves are modulated in space due to electron bunching in the position-velocity phase space. Later, this mechanism developed into the nonlinear trapping theory based on particlein-cell simulation results (Akimoto et al., 1996; Usui et al., 2005). In the nonlinear trapping theory, Langmuir waves are modulated in space due to nonlinear trapping of electrons by the electrostatic potential of the Langmuir waves. However, a recent Vlasov simulation of a very-weak-electron-beam instability has demonstrated that Langmuir waves are not directly modulated by the nonlinear trapping but are modulated by nonlinear interaction between the most unstable primary Langmuir mode and its sideband modes (Umeda, 2006). The amplitude-modulated Langmuir waves have a broadband wavenumber spectrum, which indicates a turbulent feature rather than a coherent feature. The electron phase-space distribution function of the amplitude-modulated Langmuir waves also shows a strong modification of untrapped background electrons but not any phase-space vortex associated with trapped beam electrons, implying that it is mostly the untrapped background electrons, not the trapped beam electrons, which are responsible for the wave modulations (Umeda, 2006; Silin et al., 2007).

\section{Simulation with periodic boundary}

To demonstrate whether the coherent nonlinear trapping process can really generate the spatial modulation of Langmuir waves, a one-dimensional Vlasov simulation has been carries out (Umeda, 2006). The Vlasov simulation code uses a standard time-advance scheme call the splitting scheme (Cheng and Knorr, 1976), while a conservative and non-oscillatory cubic polynomial interpolation scheme (Umeda et al., 2006) is adopted for stable time-integration of phase-space distribution functions. The simulation domain is taken along an ambient magnetic field. We assume that a very weak electron beam is drifting against the major background electrons and background ions. The density ratio of the beam component $R=n_{b} /\left(n_{e}+n_{b}\right)$ is set as $0.1 \%$, where the subscripts " $b$ " and " $e$ " represent beam electrons and background electrons, respectively. We assume that the beam and background electrons have the equal thermal velocity $V_{t e}=V_{t b}=1.0$. The total electron plasma frequency is assumed as $\omega_{p e}=1.0$. The beam drift velocity $V_{d}$ is set as $8.0 V_{t e}$. Mobile ions are also evolved in order to confirm the absence of consequences of ion dynamics on the weak-beam instability. The ion-to-electron mass ratio is set as $m_{i} / m_{e}=1836$, and the ion-to-electron temperature ratio is set as $T_{i} / T_{e}=0.1$, where the subscripts " $i$ " represents background ions. The density of ions is given by $n_{i}=n_{e}+n_{b}$. The number of spatial grid cells is $N_{x}=16384$. The number of velocity grid cells is $N_{v_{x}}=4096$ over a velocity range from $v_{\max }=24.0 V_{t e}$ to $v_{\min }=-16.0 V_{t e}$ for electrons and from $v_{\max }=13.0 V_{t i}$ to $v_{\min }=-13.0 V_{t i}$ for ions. The grid spacing is equal to $\Delta x=0.4 \lambda_{e}\left(\lambda_{e} \equiv V_{t e} / \omega_{p e}\right)$, and the time step is equal to $\omega_{p e} \Delta t=0.005$. It is noted that $\epsilon_{0}=1$ is used for simplicity. We imposed the open boundary condition in the $v_{x}$ direction and the periodic boundary condition in the $x$ direction. Here we show two simulation results; One is started with a coherent-single-wave-mode perturbation (Run A), and another is started with a white-noise perturbation (Run B).

Figure 1 shows time evolution of the wavenumber spectrum for Runs A and B. The wave intensity is normalized by $m_{e} \omega_{p e} V_{t e} / e=\sqrt{n_{e} m_{e} V_{t e}^{2}}$ and is plotted on a log scale. In Run A, an initial perturbation is imposed only at the most unstable wavenumber in order to achieve the coherent nonlinear trapping by a single sinusoidal wave mode. The most unstable primary Langmuir mode $\left(k_{x} \lambda_{e} \sim 0.144\right)$ grows from the imposed initial noise level $\left(\sim 10^{-9}\right)$, while other unstable modes grow from the round-off noise level of the doubleprecision computation $\left(\sim 10^{-15}\right)$. The primary mode saturates at $\omega_{p e} t \sim 1250$, while there appear an upper and a lower sideband mode at $k_{x} \lambda_{e} \sim 0.135$ and 0.154 , respectively, from $\omega_{p e} t \sim 2000$. Although we have started Run A with a single mode perturbation, the final wavenumber spectrum (at $\left.\omega_{p e} t=4000\right)$ has become broadband with several discrete structures.

In contrast to Run A, there appears a broadband wavenumber spectrum from $\omega_{p e} t \sim 1200$ in Run $\mathrm{B}$, because all the unstable modes grow from the same initial noise level of $\sim 10^{-9}$. Although the two simulation runs have been started with different initial wavenumber spectra, the resulting final wavenumber spectra look similar to each other. The final spectrum in Run B also shows a broadband feature with several discrete peaks at quasi-random wavenumbers. There are 
also several differences. Firstly, the most dominant mode in Run B $\left(k_{x} \lambda_{e} \sim 0.142\right)$ is not the most unstable primary mode $\left(k_{x} \lambda_{e} \sim 0.144\right)$. Secondly, the wavenumber spectra have discrete peaks at different wavenumbers. Thirdly, the generation of the sideband modes is not apparent in Run B.

The spatial modulation of Langmuir waves in Run A is likely due to the discrete structures in the wavenumber spectrum. Does the nonlinear trapping process generate the spatial modulation of Langmuir waves? To answer this question, we show the energy history of the total electric field for Run A in the top panel of Fig. 2. We also show the histories of wave intensity for primary mode $\left(k_{x} \lambda_{e}=0.144\right)$, and the most dominant two sideband modes $\left(k_{x} \lambda_{e}=0.135\right.$ and 0.154$)$ in the middle panel. The electric field amplitude and energy are normalized by $m_{e} \omega_{p e} V_{t e} / e$ and $n_{e} m_{e} V_{t e}^{2}=m_{e}^{2} \omega_{p e}^{2} V_{t e}^{2} / e^{2}$, respectively. One can see that there exist two stages in Run A. In the first stage, the primary mode linearly grows and saturate at $\omega_{p e} t \sim 1250$. After the saturation of the primary mode, the total electric field energy oscillate at $0.5 \omega_{b}$, where $\omega_{b}$ is the bounce/trapping frequency given by $\omega_{b} \equiv \sqrt{e k_{L}\left|E_{x}\left(k_{L}\right)\right| / m_{e}} \sim 0.048 \omega_{p e}$. Here $k_{L} \lambda_{e} \sim 0.144$ and $\left|E_{x}\left(k_{L}\right)\right| \sim 0.016 m_{e} \omega_{p e} V_{t e} / e$ denote the wavenumber and wave amplitude of the primary mode, respectively. However, there is no spatial modulation of Langmuir waves, implying that the spatial amplitude modulation process is different from the conventional nonlinear frequency shift by the trapping process. As seen in Fig. 2b, the growth rates of the two sideband modes slightly change after the saturation of the primary mode. This is because the velocity distribution function is modified by nonlinear trapping by the primary mode. However, the upper and lower sideband modes continue to grow linearly even after the saturation of the primary mode.

The saturated primary mode keeps almost the same amplitude until $\omega_{\text {pe }} t \sim 2000$, while the two sideband modes continuously grow. The second stage starts when the amplitude of the sideband modes reaches a certain level $\left(\sim 10^{-2.5}\right)$ at which the sideband modes can modify the velocity distribution. During the growth and saturation of the sideband modes, the primary mode becomes unstable again and is amplified. The interaction between the primary and sideband modes results in the strong spatial modulation of Langmuir waves. The spatial scale of the amplitude modulation is given by $2 \pi / \Delta k$, and the wavenumber difference between the primary and sideband modes can be estimated as $\Delta k \sim 2 \frac{\omega_{b}}{v_{p}}$ (Umeda, 2006), where $v_{p}$ denotes the phase velocity of the primary mode. In the present simulation, we can obtain a more exact value of the bounce frequency as $\omega_{b} / \omega_{p e} \sim 0.048$, because the coherent nonlinear trapping is achieved by the single mode perturbation. The wavenumber difference is obtained as $\Delta k \lambda_{e} \sim 0.01$.

Figure $2 \mathrm{a}$ and $\mathrm{b}$ suggest that the spatial modulation of Langmuir waves is not directly generated by the nonlinear trapping. However, the nonlinear trapping does play a role to
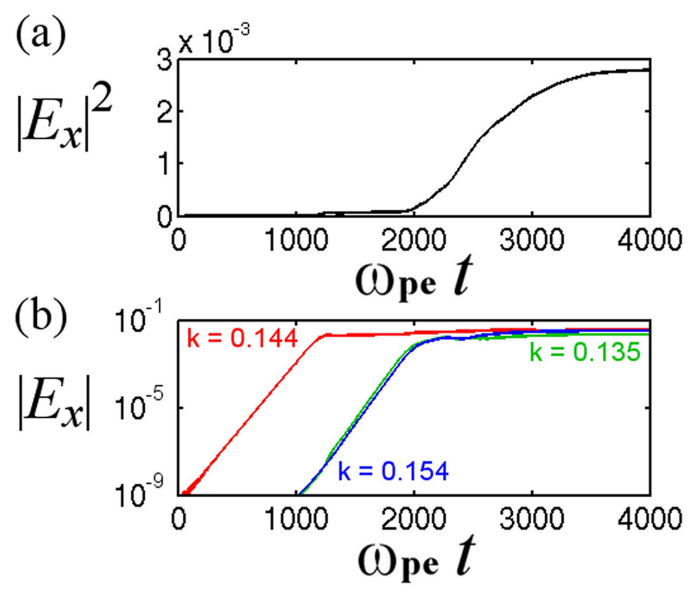

(c)

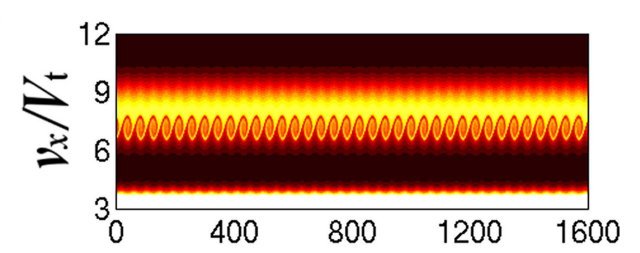

(d)

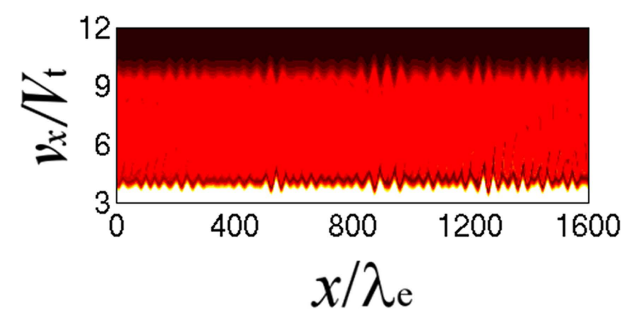

Fig. 2. Nonlinear evolution of the beam instability for Run A: (a) The energy history of the total electric field normalized by $n_{e} m_{e} V_{t e}^{2}=m_{e}^{2} \omega_{p e}^{2} V_{t e}^{2} / e^{2}$. (b) The histories of wave intensity for waves modes at $k_{x} \lambda_{e}=0.144,0.135$, and 0.154 normalized by $m_{e} \omega_{p e} V_{t e} / e$. (c) The $x-v_{x}$ phase-space electron density at $\omega_{p e} t=1250$. (d) The $x-v_{x}$ phase-space electron density at $\omega_{p e} t=3500$.

"filter" the sideband modes. It is well known that the weakelectron-beam instability is unstable over a wide wavenumber range around $k_{L}$ and that the most unstable primary Langmuir mode grows fastest and saturates earliest. By the saturation of the primary mode, there appear vortices in the position-velocity phase space of electrons as seen in the bottom panel of Fig. 2 . The velocity space around the phase velocity of the primary mode $v_{p}$ is stabilized by a strong nonlinear trapping in the phase space. As a result, unstable modes with phase velocities inside the trapping velocity range are stabilized by the nonlinear trapping, while other modes with phase velocities outside the trapping velocity range can continue to grow. In other words, the nonlinear trapping process works as a bandstop filter (Umeda, 2006). 
(a)

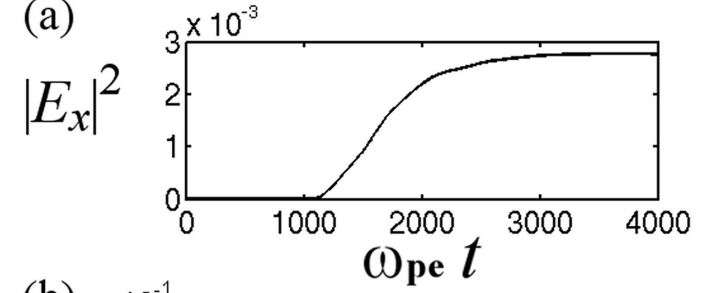

(b)

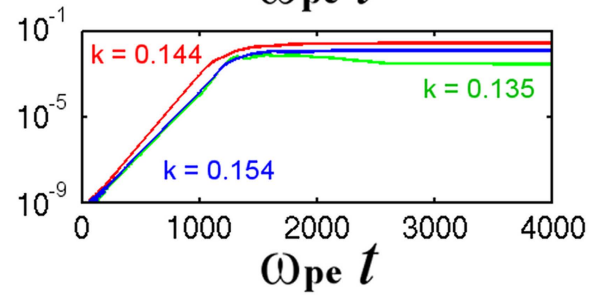

(c)

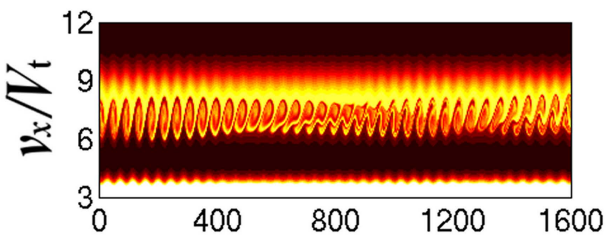

(d)

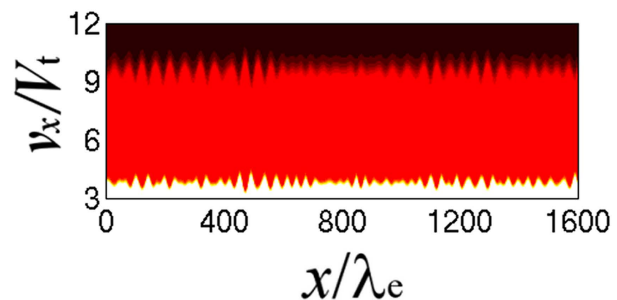

Fig. 3. Nonlinear evolution of the beam instability for Run B: (a) The energy history of the total electric field normalized by $n_{e} m_{e} V_{t e}^{2}=m_{e}^{2} \omega_{p e}^{2} V_{t e}^{2} / e^{2}$. (b) The histories of wave intensity for waves modes at $k_{x} \lambda_{e}=0.144,0.135$, and 0.154 normalized by $m_{e} \omega_{p e} V_{t e} / e$. (c) The $x-v_{x}$ phase-space electron density at $\omega_{p e} t=1250$. (d) The $x-v_{x}$ phase-space electron density at $\omega_{p e} t=3500$.

Does this story apply to the realistic case with a broadband initial noise? In Fig. 3, we show the temporal development of the weak-beam instability for Run B, in which a white noise with uniformly-distributed random phases is imposed as an initial perturbation. The histories of the total electric field, wave intensity for waves modes at $k_{x} \lambda_{e}=0.144,0.135$, and 0.154 , and the $x-v_{x}$ phase-space electron density are shown with the same format as Fig. 2. The two-step evolution of the weak-beam instability seen in Run A is not so apparent in Run B, because many wave modes are excited in a wide wavenumber range. The primary mode $\left(k_{x} \lambda_{e}=0.144\right)$ saturates earlier $\left(\omega_{p e} t \sim 1100\right)$ at a lower saturation level. The other two modes $\left(k_{x} \lambda_{e}=0.135\right.$ and 0.154$)$ also saturate at $\omega_{p e} t \sim 1100$ with the primary mode. Linearly unstable wave modes around the primary mode saturate with the primary mode in Run B. Thus the excitation of sideband modes is not identified. Note that several simulation runs with different random phases of the initial white noise are performed to examine the final wavenumber spectrum. The results show that the final wavenumber spectrum in each run has discrete peaks at different wavenumbers. This means that it depends on the initial perturbation which modes dominate in the final wavenumber spectrum.

These results show that the sideband interaction is apparent in the coherent weak-beam-plasma interaction but not in the incoherent case. Then, what is the role of coherent nonlinear trapping in the weak-beam instability with a broadband initial noise? In the bottom panel of Fig. 3, we show the position-velocity phase space of electrons at $\omega_{p e} t=1250$. It is obvious that the saturation of the primary mode involves the phase-space vortices. However, the trapping velocity range in Run B is wider than that in Run A because of the excitation of various unstable wave modes. To understand the saturation process we show in Fig. 4 the temporal development of the velocity distribution function averaged over $x$ for Run B. At $\omega_{p e} t=1000$ there is no modification of the velocity distribution function. However there exists spatial modulation of wave amplitude due to the initial white noise (not shown). At $\omega_{p e} t=1250$ we found the plateau formation in a narrow velocity range around the phase velocity of primary mode. However, it is obvious that wave modes with a phase velocity $v_{p} / V_{t e} \sim 6$ and 8 are unstable. The velocity distribution is strongly modified at $\omega_{p e} t=1500$. However, the velocity distribution is still unstable, and wave modes with a higher phase velocity can be excited at a smaller wavenumber which was not unstable in the linear stage. At $\omega_{p e} t=1750$ we found the plateau formation in a wide velocity range. However, there exists a positive gradient in the velocity distribution function, and thus wave modes with large wavenumbers $\left(v_{p} / V_{t e}<6\right)$ become unstable. Langmuir waves are excited in a broadband wavenumber range by the modification of velocity distribution function, which is similar to the sideband interaction. Thus the coherent nonlinear trapping plays a role in the generation of a turbulent (incoherent) spectrum. However, there is not any coherent nonlinear structures is the steady state as seen in Figs. $2 \mathrm{~d}$ and $3 \mathrm{~d}$. Note that a quasilinear approach taken by Silin et al. (2007) is useful to understand which mode can grow in the nonlinear stage. However, neither linear nor quasilinear approaches can tell which modes dominate in the final spectrum. In other words, the linear and quasi-linear approaches cannot tell the exact saturation level of each wave mode. The amplitude modulation of Langmuir waves is very complex involving both coherent nonlinear trapping and quasilinear modification of velocity distribution function. Thus a self-consistent simulation is a unique way to analyze the spatial scale of wave packets (e.g. Silin et al., 2007). 

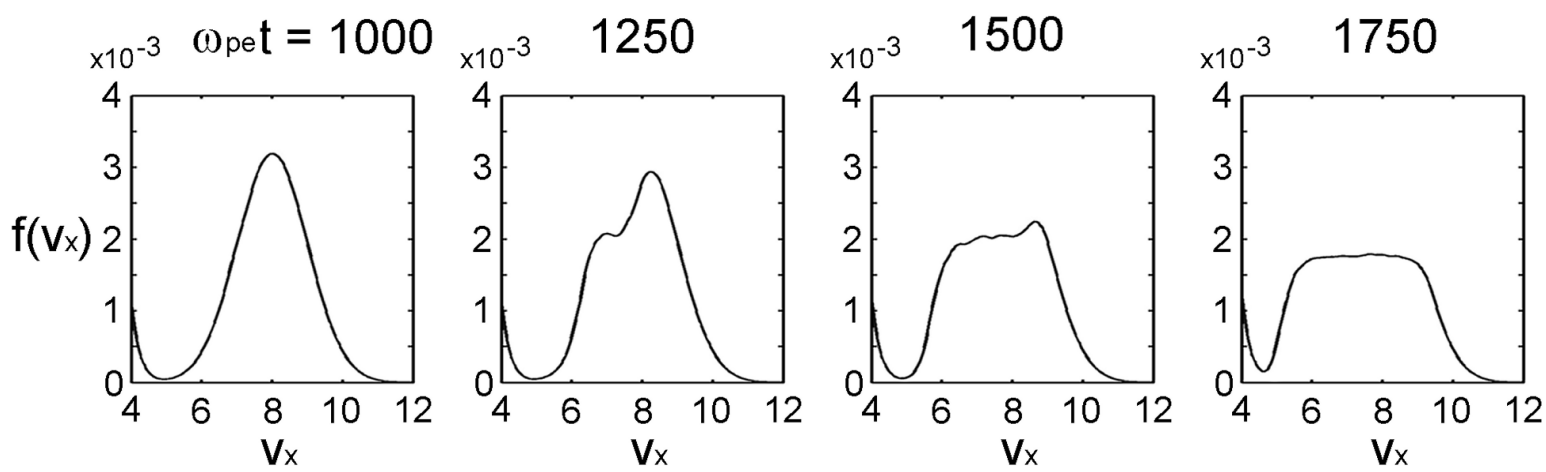

Fig. 4. Electron velocity distribution functions averaged over $x$ at different times for Run $\mathrm{B}$. The velocity is normalized by $V_{t e}$. The distribution functions are normalized to unity.

\section{Simulation with open boundary}

We extend the one-dimensional simulation in the previous section to a more realistic model (Run C). We adopted a one-dimensional open system in which an electron beam is injected into the system from a boundary while outgoing plasmas are absorbed without reflection at boundaries (e.g. Umeda et al., 2002). As the initial condition, we assume that the background plasma exists uniformly in the simulation domain without the electron beam. When a computer simulation is started, the electron beam is continuously injected from the left boundary into the background homogeneous plasma. The injected electron beam and the background electrons form an unstable velocity distribution function. In the present study, we inject the electron beam with a constant flux. The background electrons and ions are assumed to be continuous at the right boundary as if there is no boundary. The background ions are also assumed to be continuous at the left boundary, while the flux of background electrons at the left boundary is modified to keep the charge neutrality. We used the same simulation parameters as Run B to make a direct comparison between the periodic and open systems.

The right panel of Fig. 5 shows the energy density of electric field $\left|E_{x}(x, t)\right|^{2}$ for Run C. In the left and middle panels, plots of the energy density of electric field for Runs A and $\mathrm{B}$ are also shown as references. The magnitude is normalized by $n_{e} m_{e} V_{t e}^{2}=m_{e}^{2} \omega_{p e}^{2} V_{t e}^{2} / e^{2}$. Note that the entire simulation domain is not shown in Fig. 5. In the present open system where a very weak electron beam is continuously injected from the boundary, the beam instability develops in both space and time (e.g. Umeda et al., 2002). Initially, the electron beam is injected into the unperturbed plasma, making the velocity distribution function unstable. However, the sudden injection of the electron beam at the onset also generates an impulsive strong perturbation at a high level of $\sim 10^{-6}$, which is a much higher level than that of the initial perturbation $\left(\sim 10^{-10}\right)$. Thus the weak-beam instability in Run C saturates earlier (at $\omega_{p e} t \sim 600$ ) than in Run B.
There also exist other differences. In Runs A and B, wave packets are observed in an almost random manner in both space and time. The temporal waveform of the Langmuir wave packets at $x / \lambda_{e} \sim 2000$ in Run $\mathrm{C}$ is similar to that in Run B. On the other hand, the temporal waveform of the Langmuir wave packets at $x / \lambda_{e}=500 \sim 1000$ becomes quasiperiodic from $\omega_{p e} t \sim 2400$ in Run C. The period of the generation of wave packets is $t \sim 450 / \omega_{p e}$, which is longer than the ion plasma period, i.e., $t=2 \pi \sqrt{m_{i} / m_{e}} / \omega_{p e} \sim 270 / \omega_{p e}$, implying the absence of ion dynamics. The nonlinear beamplasma interaction takes place uniformly in space in Runs $\mathrm{A}$ and $\mathrm{B}$, while the nonlinear interaction results in the formation of a single Langmuir wave packet in a localized region close to the beam source $\left(x / \lambda_{e} \sim 500\right)$ in Run C. The wave packet propagates at the group velocity of the primary Langmuir mode, which is much slower than the beam velocity. Since the electron beam is continuously injected in Run C, the free energy source is supplied to Langmuir waves and positive gradient in the velocity distribution exists for a longer time. Thus the amplitude of excited Langmuir mode in Run $\mathrm{C}$ becomes much higher than in Runs A and B.

Figure 6 shows time evolution of the wavenumber spectrum for Run C. The wave intensity is normalized by $m_{e} \omega_{p e} V_{t e} / e=\sqrt{n_{e} m_{e} V_{t e}^{2}}$ and is plotted on a log scale. We found several discrete peaks at $k_{x} \lambda_{e} \sim 0.125,0.144$, and 0.154 in the wavenumber spectrum at $\omega_{p e} t \sim 600$. The saturation level of these modes is $\sim 10^{-2}$. Although this saturation level is close to the saturation level in the runs with periodic boundaries (Runs A and B), the wave amplitude in Runs $\mathrm{C}$ and $\mathrm{B}$ (or $\mathrm{A}$ ) is very different. The Langmuir waves are uniformly excited in Runs A and B, while a single wave packet is excited in a localized region in Run C. Since the Fourier transformation of a spatially-localized wave packet is taken in Fig. 6, the actual amplitude of the wave packet is much higher $\left(\sim 0.3 m_{e} \omega_{p e} V_{t e} / e\right)$.

The discrete structure in the wavenumber spectrum in Run $\mathrm{C}$ is similar to that in the run with a uniform and coherent initial perturbation (Run A), which is likely because the co- 

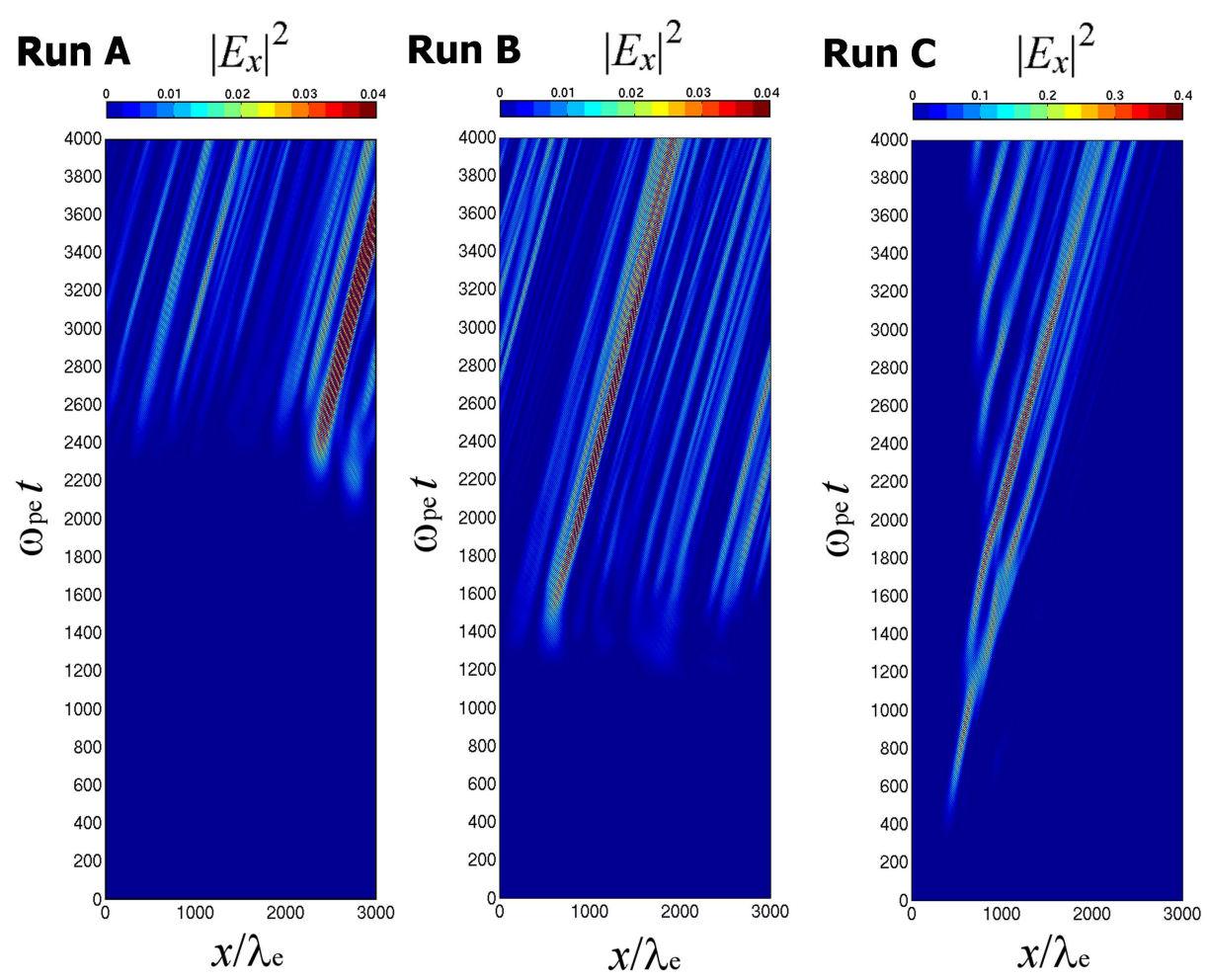

Fig. 5. Electric field energy density as a function of time and position for Runs (left) A , (middle) B, and (right) C. The electric field energy density is normalized by $n_{e} m_{e} V_{t e}^{2}$.

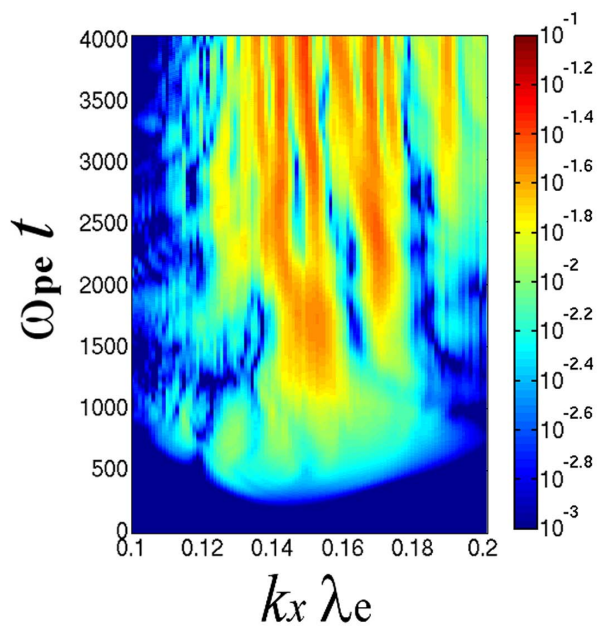

Fig. 6. Time evolution of the wavenumber spectrum for Run C. The wave intensity is normalized by $m_{e} \omega_{p e} V_{t e} / e$.

herent behavior of the beam-plasma interaction is enhanced by a high noise level $\left(\sim 10^{-6}\right)$ due to the sudden injection of electron beam. However, these modes are not dominant for $\omega_{p e} t>1000$. Other wave modes with a broader wavenumber band appear in $k_{x} \lambda_{e}=0.14 \sim 0.16$ at $\omega_{p e} t=1200$. This may correspond to the break of the first wave packet and the formation of the second packet in the left panel of Fig. 5. The amplitude of the wave packet exceeds $0.6 m_{e} \omega_{p e} V_{t e} / e$ but is lower than $1.0 m_{e} \omega_{p e} V_{t e} / e$, implying that the parametric instabilities due to ion dynamics are not essential. The two-step evolution of the weak-beam instability in Run C is similar to that in Run A.

For $\omega_{p e} t>2000$ we found three discrete peaks in the wavenumber spectrum. It is unclear whether these are an artifact of the present boundary condition or a real physics. However, a typical wavenumber difference of the discrete structure is about $\Delta k \lambda_{e} \sim 0.023$ at $\omega_{p e} t=2500$, which corresponds to the spatial scale length of wave packets $L / \lambda_{e} \sim 270$. From Fig. 5 the group velocity of the wave packets is estimated as $v_{g} \sim 0.6 V_{t e}$. Thus a typical timescale of amplitude modulation is estimated as $t=L / v_{g} \sim 450 / \omega_{p e}$, which is consistent with the time interval of the wave packets at $x / \lambda_{e}=500 \sim 1000$. For $\omega_{p e} t>3000$ we also found several discrete structures in the wavenumber spectrum. These structures are expected to be the incoherent behavior of the beamplasma interaction as seen in Run B.

In order to analyze the effect of coherent nonlinear trapping process, phase-space distribution functions of electron at different times are shown in Fig. 7. At all the time there exist coherent electron phase-space vortices in the region close to the beam source, which is different from Run B, in which 
electron phase-space vortices modulated by an initial perturbation appear only at the saturation state (see Fig. 3c). The effect of the initial perturbation is likely suppressed by the spatial development of the beam-plasma instability, because an envelope of a wave packet is formed by the spatial development. It is also noted that the size of vortices in Run C becomes much larger than that in Runs A and B (see Figs. 2c and 3c).

At $\omega_{p e} t=600$ we found electron phase-space vortices with propagation velocities $v_{p} / V_{t} \sim 6$ and 8 for $x / \lambda_{e}=600 \sim 800$. These vortices correspond to the wavenumber enhancement at $k_{x} \lambda_{e} \sim 0.125$ and 0.155 , implying that there is a process similar to the sideband interaction as seen in Run A. At $\omega_{p e} t=1200$ there are very large vortices for $x / \lambda_{e}=600 \sim 800$. Later these vortices split into two wave packets as seen in the left panel of Fig. 5. At $\omega_{p e} t=2000$ we found small vortices with a slow propagation velocity $\left(v_{p} / V_{t} \sim 5\right)$ between the first and second packets (at $\left.x / \lambda_{e} \sim 1100\right)$. Such vortices are also found at $\omega_{p e} t=2500$ and $x / \lambda_{e} \sim 1000$, suggesting that the quasi-periodic amplitude modulation would be generated by the interaction between the primary Langmuir mode and wave modes with a slow phase velocity $\left(v_{p} / V_{t} \sim 5\right)$. It is also noted that the phase-space distribution function for $x / \lambda_{e}=800 \sim 1000$ is different from that for $x / \lambda_{e}>1200$ where the phase-space distribution function shows a more turbulent feature. In other words, the incoherent process is dominant for $x / \lambda_{e}>1200$, while the coherent nonlinear trapping process is likely dominant in the region close to the beam source. For $x / \lambda_{e}=800 \sim 1000$, Langmuir wave packets are formed by the interaction between two or three wave modes with different phase velocities, which is similar to the sideband interaction.

\section{Conclusion and discussion}

In this paper we have briefly reviewed mechanisms for the amplitude modulation and packet formation of Langmuir waves by performing one-dimensional Vlasov simulations with different initial and boundary conditions.

In the run with a uniform and coherent initial perturbation, there are two stages for the nonlinear development of the weak-electron-beam instability. In the first stage, a weak-electron-beam instability excites Langmuir waves over a wide wavenumber range which is essentially a linear process. The primary Langmuir mode saturates by the nonlinear trapping of electrons, which involves electron-phase-space vortices. The nonlinear trapping process filters the unstable modes with phase velocities inside the trapping velocity range. In the second stage, the sideband modes grow to a level comparable to the saturation level of the primary mode. The sideband modes modify the phase-space distribution function, making the primary mode unstable again. As a result, the primary mode and the sideband modes dominate. Langmuir waves are modulated in space by an interaction

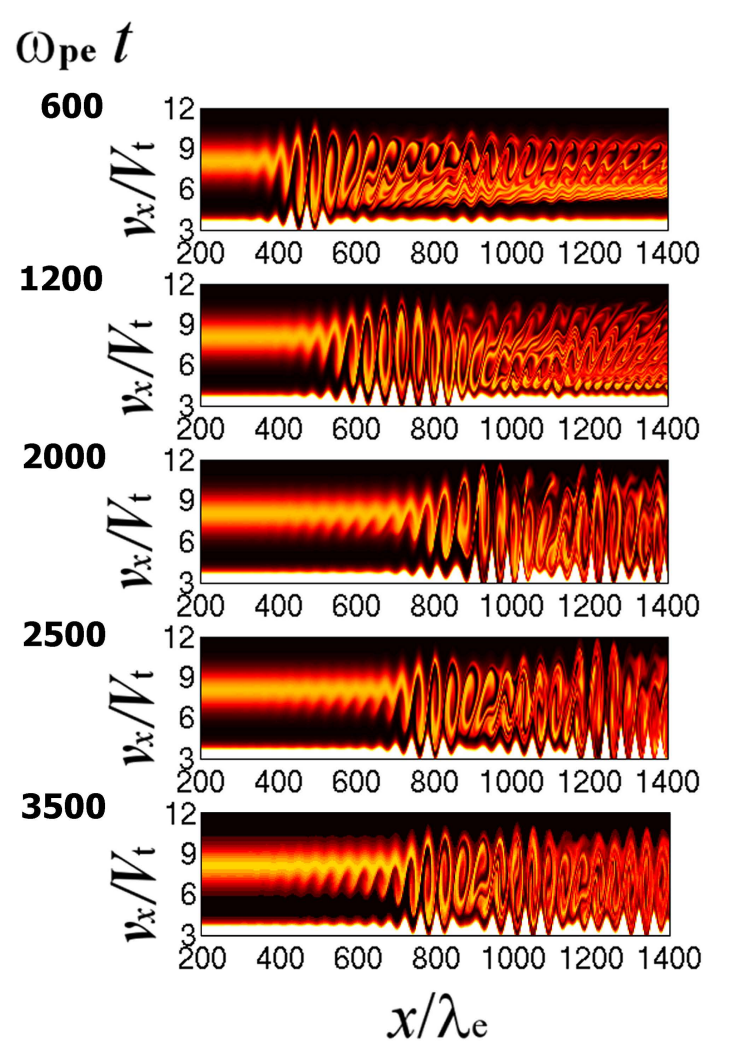

Fig. 7. The $x-v_{x}$ phase-space electron density at $\omega_{p e} t=600$, 1200, 2000, 2500, and 3500 .

between the primary and sideband modes. The sideband interaction is an extension of the kinetic localization Muschietti et al. $(1995,1996)$ and is a modification of the nonlinear trapping theory (Akimoto et al., 1996; Usui et al., 2005). The nonlinear trapping process does not directly generate the spatial modulation of Langmuir waves, but plays a role to filter wave modes with wavenumbers close to the most unstable wave number. A turbulent wavenumber spectrum with several discrete peaks results in the spatial modulation of Langmuir waves. In contrast, the coherent process is not apparent in the run with an incoherent white-noise perturbation. A quasilinear modification of velocity distribution functions is more essential than in the run with the coherent initial perturbation (Silin et al., 2007).

In the run with open boundaries where an electron beam is injected from a boundary, nonlinear evolution of the beamplasma instability is drastically modified from that of the run with periodic boundaries. The coherent nonlinear trapping process is enhanced by the localized injection of an electron beam. The result again looks similar to that of the run with uniform and coherent initial perturbation. Because of the spatial development of the beam-plasma instability, the saturation level also changes. The result suggests that beamplasma interactions in nonuniform systems become differ- 
ent from the temporal evolution in uniform periodic systems when the propagation velocity of waves and free-energy source is different.

In conclusion, both coherent nonlinear process and incoherent turbulent process are important for the formation of Langmuir wave packets. In the present study we have also solved ion distributions. However, the effect of ion dynamics is absent, because the amplitude of Langmuir wave packets is smaller than $1.0 m_{e} \omega_{p e} V_{t e} / e$. Simulation runs with a high-density electron beam is left as a future work, in which parametric instabilities due to ion dynamics would play an important role in the formation of Langmuir wave packets.

Acknowledgements. This work was supported by Grant-in-Aid for Young Scientists (Start-up) \#19840024 and in part by Grant-in-Aid for Creative Scientific Research \#17GS0208 "The Basic Study of Space Weather Prediction" from the Ministry of Education, Science, Sports, Technology, and Culture of Japan. The computer simulations were performed on the Fujitsu PRIMEPOWER HPC 2500 at Kyoto University and Nagoya University. The computing resources are provided by Research Institute for Sustainable $\mathrm{Hu}$ manosphere, Kyoto University and Solar-Terrestrial Environment Laboratory, Nagoya University.

Edited by: A. C. L. Chian

Reviewed by: D. L. Newman and another anonymous referee

\section{References}

Akimoto, K., Omura, Y., and Matsumoto, H.: Rapid generation of Langmuir wave packets during electron beam-plasma instabilities, Phys. Plasmas, 3, 2559-2563, 1996.

Bale, S. D., Burgess, D., Kellogg, P. J., Goetz, K., Howard, R. L., and Monson, S. J.: Phase coupling in Langmuir wave packets: Possible evidence of three-wave interactions in the upstream solar wind, Geophys. Res. Lett., 23, 109-112, 1996.

Berk, H. L. and Roberts, K. V.: Nonlinear study of Vlasov's equation for a special class of distribution functions, Phys. Fluids 10, 1595-1597, 1967.

Bonnell, J., Kintner, P., Wahlund, J.-E., and Holtet, J. A.: Modulated Langmuir waves: Observations from Freja and SCIFER, J. Geophys. Res., 102, 17 233-17 240, 1997.

Cairns, I. H. and Robinson, P. A.: Theory for low-frequency modulated Langmuir wave packets, Geophys. Res. Lett., 19, $2187-$ 2190, 1992.

Cairns, I. H., Robinson, P. A. and Smith, N. I.: Arguments against modulational instabilities of Langmuir waves in Earth's foreshock, J. Geophys. Res., 103, 287-298, 1998.

Cheng, C. Z., and Knorr, G.: The integration of the Vlasov equation in configuration space, J. Comput. Phys., 22, 330-351, 1976.

Ergun, R. E., Carlson, C. W., McFadden, J. P., Clemmons, J. H., and Boehm, M. H.: Evidence of a transverse Langmuir modulational instability in a space plasma, Geophys. Res. Lett., 18, 1177-1180, 1991.

Gaelzer, R., Yoon, P. H., Umeda, T., Omura, Y., and Matsumoto, H.: Harmonic Langmuir waves. II. Turbulence spectrum, Phys, Plasmas, 10, 373-381, 2003.
Gurnett, D. A., Hospodarsky, G. V., Kurth, W. S., Williams, D. J., and Bolton, S. J.: Fine structure of Langmuir waves produced by a solar electron event, J. Geophys. Res., 98, 5631-5638, 1993.

Kellogg, P. J., Monson, S. J., Goetz, K., Howard, R. L. Bougeret, J. L., and Kaiser, M. L.: Early wind observations of bow shock and foreshock waves, Geophys. Res. Lett., 23, 1243-1246, 1996.

Kellogg, P. J., Goetz, K., Monson, S. J., and Bale, S. D.: A search for Langmuir solitons in the Earth's foreshock, J. Geophys. Res., 104, 6751-6758, 1999a.

Kellogg, P. J., Goetz, K., Monson, S. J., and Bale, S. D.: Langmuir waves in a fluctuating solar wind, J. Geophys. Res., 104, 17069 17 078, 1999b.

Kojima, H., Furuya, H.. Usui, H., and Matsumoto, H.: Modulated electron plasma waves observed in the tail lobe: Geotail waveform observations, Geophys. Res. Lett., 24, 3049-3052, 1997.

Lizunov, G. V., Khotyaintsev, Y., and Stasiewicz, K.: Parametric decay to lower hybrid waves as a source of modulated Langmuir waves in the topside ionosphere, J. Geophys. Res., 106, 24755 24764, 2001

Matsukiyo, S., Treumann, R. A., and Scholer, M.: Coherent waveforms in the auroral upward current region, J. Geophys. Res., 109, A06212, doi:10.1029/2004JA010477, 2004.

Matsumoto, H., Kojima, H., Miyatake, T., Omura, Y., Okada, M., Nagano, I., and Tsutsui, M.: Electrostatic solitary waves (ESW) in the magnetotail: BEN wave forms observed by Geotail, Geophys. Res. Lett., 21, 2915-2918, 1994.

Muschietti, L., Roth, I., and Ergun, R. E.: Kinetic localization of beam-driven Langmuir waves, J. Geophys. Res., 100, 17481 $17490,1995$.

Muschietti, L., Roth, I., and Ergun, R. E.: On the formation of wave packets in planetary foreshocks, J. Geophys. Res., 101, 15605 $15613,1996$.

Pottelette, R., Ergun, R. E., Treumann, R. A., Berthomier, M., Carlson, C. W., McFadden, J. P., and Roth, I.: Modulated electronacoustic waves in auroral density cavities: FAST observations, Geophys. Res., Lett., 26, 2629-2632, 1999.

Silin, I., Sydora, R., and Sauer, K.: Electron beam-plasma interaction: Linear theory and Vlasov-Poisson simulations, Phys. Plasmas, 14, 012106, 2007.

Soucek, J. Krasnoselskikh, V., Dudok de Wit, T., Pickett, J., and Kletzing, C.: Nonlinear decay of foreshock Langmuir waves in the presence of plasma inhomogeneities: Theory and Cluster observations, J. Geophys. Res., 110, A08102, doi:10.1029/2004JA010977 2005.

Stasiewicz, K., Holback, B., Krasnoselskikh, V., Boehm, M., Bostrom, R., and Kintner, P. M.: Parametric instabilities of Langmuir waves observed by Freja, J. Geophys. Res., 101, 21515 $21526,1996$.

Umeda, T., Omura, Y., Matsumoto, H., and Usui, H.: Formation of electrostatic solitary waves in space plasmas: Particle simulations with open boundary conditions, J. Geophys. Res., 107, 1449, doi:10.1029/2001JA000286, 2002.

Umeda, T., Omura, Y., Yoon, P. H., Gaelzer, R., and Matsumoto, H.: Harmonic Langmuir waves. III. Vlasov simulation, Phys. Plasmas, 10, 382-391, 2003.

Umeda, T., Ashour-Abdalla, H., and Schriver, D.: Comparison of numerical interpolation schemes for one-dimensional electrostatic Vlasov code, J. Plasma Phys., 72, 1057-1060, 2006.

Umeda, T.: Vlasov simulation of amplitude-modulated Langmuir 
waves, Phys. Plasmas, 13, 092304, 2006.

Usui, H., Furuya, H., Kojima, H., Matsumoto, H., and Omura, Y.: Computer experiments of amplitude-modulated Langmuir waves: Application to the Geotail observation, J. Geophys. Res., 110, A06203, doi:10.1029/2004JA010703, 2005.
Yoon, P. H., Gaelzer, R., Umeda, T., Omura, Y., and Matsumoto, H.: Harmonic Langmuir waves. I. Nonlinear dispersion relation, Phys. Plasmas, 10, 364-372, 2003. 